\title{
Rogue waves and periodic solutions of a nonlocal nonlinear Schrödinger model
}

\author{
C. B. Ward,${ }^{1}$ P. G. Kevrekidis, ${ }^{1,2}$ T. P. Horikis $\odot,{ }^{3}$ and D. J. Frantzeskakis $\odot^{4}$ \\ ${ }^{1}$ Department of Mathematics and Statistics, University of Massachusetts, Amherst, Massachusetts 01003-4515, USA \\ ${ }^{2}$ Mathematical Institute, University of Oxford, Oxford, OX2 6GG, United Kingdom \\ ${ }^{3}$ Department of Mathematics, University of Ioannina, Ioannina 45110, Greece \\ ${ }^{4}$ Department of Physics, National and Kapodistrian University of Athens, Panepistimiopolis, Zografos, Athens 15784, Greece
}

(Received 15 October 2019; accepted 11 February 2020; published 20 March 2020)

\begin{abstract}
In the present paper, a nonlocal nonlinear Schrödinger (NLS) model is studied by means of a recent technique that identifies solutions of partial differential equations by considering them as fixed points in space-time. This methodology allows us to perform a continuation of well-known solutions of the local NLS model to the nonlocal case. Four different examples of this type are presented, namely, (a) the rogue wave in the form of the Peregrine soliton and (b) the generalization thereof in the form of the Kuznetsov-Ma breather, as well as two spatiotemporally periodic solutions in the form of elliptic functions. Importantly, all four wave forms can be continued in intervals of the parameter controlling the nonlocality of the model. The first two can be continued in a narrower interval, while the periodic ones can be extended to arbitrary nonlocalities and, in fact, present an intriguing bifurcation whereby they merge with (only) spatially periodic structures. The results suggest the generic relevance of rogue waves and related structures, as well as periodic solutions, in nonlocal NLS models.
\end{abstract}

DOI: 10.1103/PhysRevResearch.2.013351

\section{INTRODUCTION}

The study of dispersive media exhibiting a nonlocal nonlinear response is a subject that is enjoying increasing attention over the past few years [1-3]. This is mainly due to the fact that relevant models and their solutions, especially of the nonlinear Schrödinger (NLS) variety, emerge in a wide range of physical contexts. These range from thermal optical media $[1,4]$ and nematic liquid crystals $[2,3,5]$ and from plasmas $[6,7]$ to water waves $[8,9]$ and dipolar Bose-Einstein condensates $[10,11]$. In addition, there are certain settings with a local nonlinearity, where an effective nonlocal description is particularly relevant as, e.g., in the case of quadratic nonlinear media [12]. In this case, bright and dark soliton solutions, as well as other applications, such as the prediction of regimes for quadratic soliton pulse compression [13,14], and the existence of $X$ waves [15], have been discussed. It is, thus, naturally of interest to explore the different types of solitary wave solutions that may arise in nonlocal NLS systems, and how nonlocality may alter the properties and behavior of media with a local nonlinear response [16-18].

Another topic of wide interest during the past decade has been the study of rogue (freak) waves, which are structures of large amplitude that can roughly be divided into (a) the ones that "appear spontaneously out of nowhere and disappear without a trace" [19] and (b) the ones that are generated gradually through energy transfer in multiple soliton colli-

Published by the American Physical Society under the terms of the Creative Commons Attribution 4.0 International license. Further distribution of this work must maintain attribution to the author $(s)$ and the published article's title, journal citation, and DOI. sions. The latter type of rogue waves have lifetimes relevant for biological processes (e.g., DNA denaturation [20]), and supercontinuum laser light sources [21]. Rogue waves have been explored mainly in hydrodynamics [22-24], but also in numerous other areas. These include, but are not limited to, nonlinear optics [25-31], superfluid helium [32], as well as plasmas [33]. These multifaceted experimental studies have, in turn, triggered a wide range of theoretical explorations which by now have been summarized in a series of reviews [34-40], but also importantly in a series of books on this research theme [41-44].

In the present paper, we combine these two cutting edge themes by exploring rogue waves and related coherent structures in nonlocal media motivated by the above optical, liquid crystal, and water wave applications. It should be noted that a search of the literature of rogue waves in nonlocal NLS models will yield a number of results, such as, e.g., Ref. [45]. However, these concern a mathematically motivated (via $\mathcal{P} \mathcal{T}$-symmetry and related considerations) nonlocal variant of the NLS [46]. There is a paucity of results concerning rogue waves of physically relevant nonlocal NLS models, such as, e.g., the one considered in Ref. [47]. However, this is rather understandable given the nonintegrability of such models and the distinct lack of tools for tackling rogue waves beyond the integrable limit and its associated techniques, such as the inverse scattering method.

Here, we aim to provide a number of results regarding the model of the nonlocal NLS that is of wide relevance to applications. Our approach leverages a recently proposed technique reported in Ref. [48]. In this work, it was recognized that it is difficult to identify rogue waves (contrary to what is the case with solitons), due to their nonstationary nature in time. However, considering them in space-time, i.e., treating time as a spatial direction, one realizes that rogue 




(a)

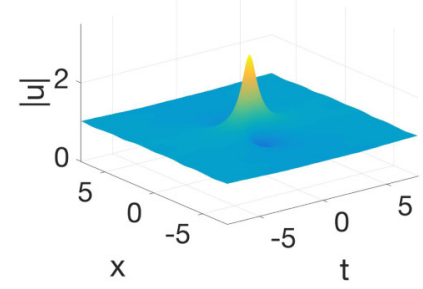

(b)

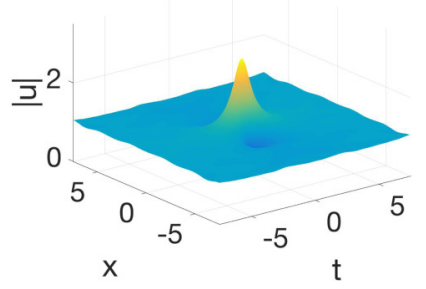

(c)

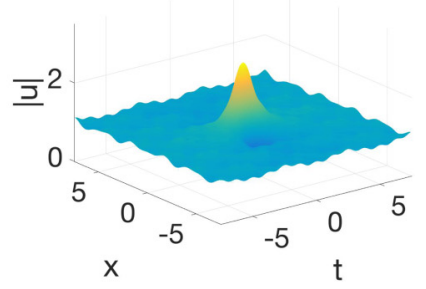

(d)

FIG. 1. Continuation over increasing $v$ values of the Peregrine rogue wave solution of the NLS. The modulus of the wave function is shown as a function of space $x$ and time $t$, once the iterative identification of the solution converges. Values of $v$ used in each of the panels: (a) 0 , (b) 0.05 , (c) 0.15 , (d) 0.2 .

waves are localized solutions in that setting. Thus, one canin principle-construct fixed-point methods (based, e.g., on a conjugate gradient (CG) based variant of the Newton method [48]) that will converge to such wave structures and identify them as numerically exact solutions. To be more specific, we have discretized the space and time derivatives using the fast Fourier transform and then used a Newton conjugate gradient (Newton-CG) algorithm to solve the resulting equations. A key advantage of such a methodology is that it does not hinge in any critical way on integrability and, indeed, starting from the integrable limit it can be used in a variety of nonintegrable settings, such as the nonlocal one that we consider here. It is this tool that will permit us to converge to the rogue wave (in the form of the Peregrine soliton) for a range of parameter values of the nonlocality parameter, referred to as $v$ below in our model. In addition to the prototypical Peregrine structure, we will also seek its periodic - in the propagation variablegeneralization, namely the Kuznetsov-Ma (KM) breather. Both of these will be found to be possible to continue within a certain interval of the nonlocality parameter (roughly up to $v=0.2$ ). Apart from these structures, we will also consider and identify additional states that our analysis can reveal in the nonlocal system, namely, periodic states in space-time (starting from the elliptic function local limit). We will see that these can be continued for essentially arbitrary $v$, yet they feature a bifurcation becoming stationary (or independent of the propagation direction) beyond a certain critical threshold.

Our presentation will be structured as follows. In Sec. II, we will present the solutions of interest in the local limit of the regular NLS model (i.e., for $v=0$ ). In Sec. III, we will present the numerical extensions of the solutions via the above method to the nonlocal case of finite $v$. Finally, in Sec. IV, we will summarize our findings and present our conclusions.

\section{THE MODEL AND ITS LOCAL LIMIT SOLUTIONS}

We consider a nonlocal variant of the NLS model, which, in dimensionless form, can be expressed as $[2,3,47]$

$$
\begin{gathered}
i \frac{\partial u}{\partial t}+\frac{1}{2} \frac{\partial^{2} u}{\partial x^{2}}+\theta u-\mu u=0, \\
\nu \frac{\partial^{2} \theta}{\partial x^{2}}-2 q \theta=-2|u|^{2},
\end{gathered}
$$

where $u=u(x, t), \theta=\theta(x, t)$, and, in the context of nematic liquid crystals, $t$ plays the role of the propagation coordinate. The dependent variable $u$ is the complex valued, slowly varying envelope of the optical (electric) field, and $\theta$ is the optically induced deviation of the director angle. The nonlocality parameter $v$ measures the strength of the response of the nematic in space, with a highly nonlocal response corresponding to $v$ large. Notice that in the nonlocal regime with $v$ large, the optically induced rotation $\theta$ is small; on the other hand, the standard NLS equation with the local Kerr nonlinearity is recovered when $v=0$. The parameter $q$ is related to the square of the applied static field which pretilts the nematic dielectric, while $\mu$ plays the role of the propagation constant. Obviously, inclusion of the term $\mu u$ in Eq. (1) (which is normally omitted) offers a constant background where certain types of solutions (such as the Peregrine soliton-see below) can exist, and is trivially removed via a constant phase transformation. Hereafter, we set $q=1$.

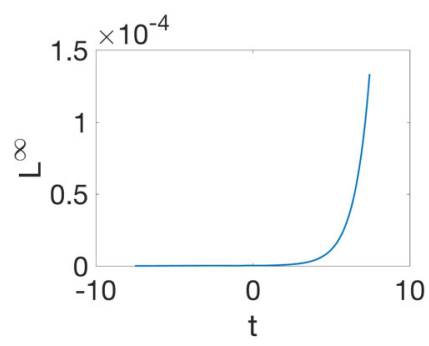

(a)

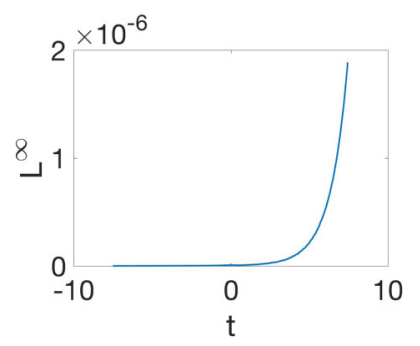

(b)



(c)

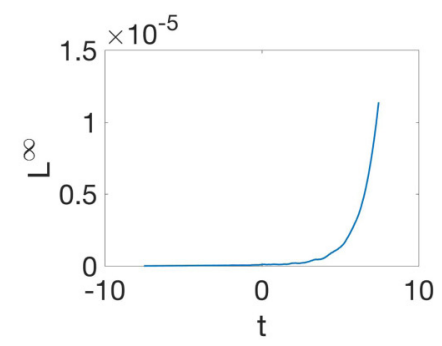

(d)

FIG. 2. $L^{\infty}$ error (i.e., norm of the difference) between the numerical ETDRK4 solution of Eqs. (1) and (2) and the converged Peregrine soliton wave form obtained by the Newton-CG method for the different case examples of nonlocality parameter $v$ shown in Fig. 1 . Values of $v$ used in each of the panels: (a) 0 , (b) 0.1 , (c) 0.15 , (d) 0.2 . 


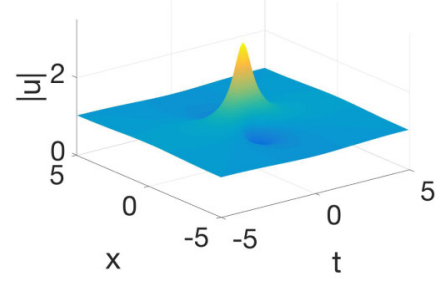

(a)

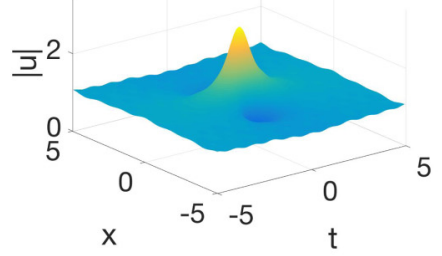

(b)

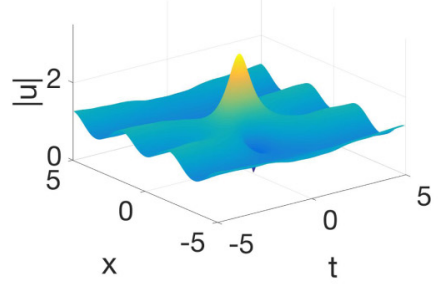

(c)

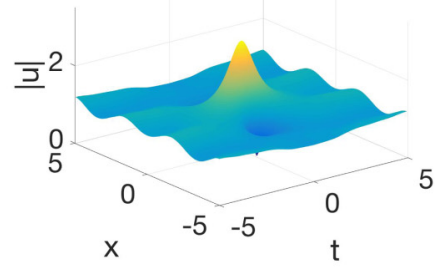

(d)

FIG. 3. Continuation over increasing $v$ values of the KM breather solution of the NLS. Note that the solution seems to transition into a breather on top of a periodic background as the nonlocality parameter is increased. Values of $v$ used in each of the panels: (a) 0 , (b) 0.1 , (c) 0.15, (d) 0.2 .

The system Eqs. (1) and (2) can be considered as a single integro-differential equation provided Fourier transforms are utilized. This will not limit our set of solutions however, as we will make only numerical use of the transform pair. As such, a periodic domain is used to integrate the system in $x$ and nondecaying functions will not generate problems with the transform not converging. Thus, using the Fourier transform, we can rewrite Eq. (2) as

$$
\theta=\mathcal{F}^{-1}\left[\frac{\mathcal{F}\left[2|u|^{2}\right]}{v k^{2}+2}\right]
$$

where $k$ is the wave number (the relevant Fourier variable). Plugging this into Eq. (1) yields a nonlocal equation in $u$ alone and will be the equation with which we work in our numerical computations hereafter. Equation (3) implies that the kernel in the equivalent integro-differential equation is of the form $\exp (-|x|)$. This corresponds to the focusing case with $q=1$ (which we consider here), while in the defocusing case with $q=-1$ the kernel is of the form $\sin (|x|)$, which is of particular importance in other settings [49]. Note that many other types of response functions have also been considered for nonlocal NLS types of equations, and it has been attempted to define generic properties of the types of response functions [50].

The four solutions that will be of interest hereafter are analytically available in the NLS limit $(v=0)$. The first one is the famous Peregrine soliton [51] which, for $\mu=1$, reads

$$
u(x, t)=1-\frac{2(1+4 i t)}{1+4 x^{2}+4 t^{2}} .
$$

This structure has been the subject of numerous recent experimental observations in hydrodynamics [22], nonlinear optics [26], plasmas [33], and so on.

The second one is the periodic generalization of the Peregrine soliton in the evolution direction, namely, the so-called KM breather given by $[52,53]$

$$
u(x, t)=1-\frac{2\left(b^{2}-1\right) \cos \left(2 b \sqrt{b^{2}-1} t\right)+i 2 b \sqrt{b^{2}-1} \sin \left(2 b \sqrt{b^{2}-1} t\right)}{b \cosh \left(2 \sqrt{b^{2}-1} x\right)-\cos \left(2 b \sqrt{b^{2}-1} t\right)},
$$

where $b$ is an arbitrary parameter (with $b>1$ ). It is worthwhile to note that this solution has been experimentally observed as well [27].

We now consider some spatiotemporally periodic solutions stemming from the classic work of Ref. [54], both of them for simplicity given for $\mu=1 / 2$. The first is a doubly periodic solution given by

$$
\begin{gathered}
u(x, t)=\frac{\kappa}{2} \frac{A(x) \operatorname{cn}(t / 2, \kappa)+i \sqrt{1+\kappa} \operatorname{sn}(t, \kappa)}{\sqrt{1+\kappa}-A(x) \operatorname{dn}(t / 2, \kappa)}, \\
A(x)=\operatorname{cd}\left(\sqrt{\frac{1+\kappa}{2}} x, \sqrt{\frac{1-\kappa}{1+\kappa}}\right),
\end{gathered}
$$

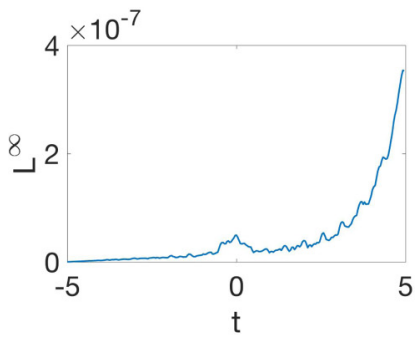

(a)

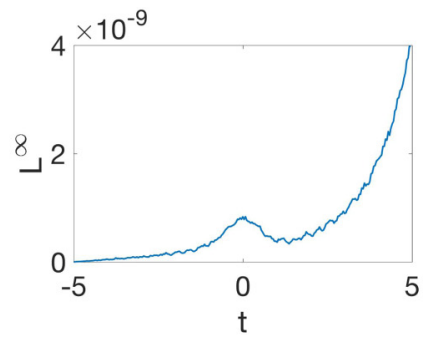

(b)



(c)

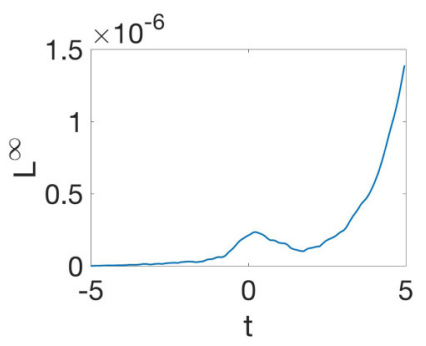

(d)

FIG. 4. $L^{\infty}$ error between the solution obtained by the Newton-CG method (as shown in Fig. 3) verus the solution obtained via the ETDRK4 method when the KM breather profile is seeded into the latter as an initial condition. Values of $v$ used in each of the panels: (a) 0, (b) 0.1, (c) 0.15 , (d) 0.2 . 




(a)



(b)

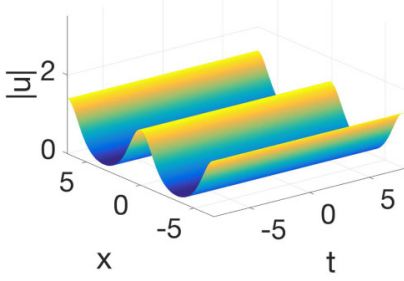

(c)

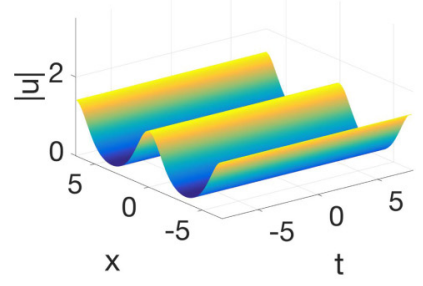

(d)

FIG. 5. Continuation over increasing $v$ values of the doubly periodic first solution of the NLS. It is seen that the doubly periodic solution bifurcates into a solution whose (modulus) profile is stationary and thus time-independent as $v$ is increased. Values of $v$ used in each of the panels: (a) 0 , (b) 0.5 , (c) 1 , (d) 1.5 .

where $\kappa$ is an arbitrary parameter (with $0<\kappa<1$ ). The second is another doubly periodic solution given by

$$
u(x, t)=\frac{\sqrt{\frac{\kappa}{1+\kappa}} \operatorname{cn}\left(\frac{x}{\sqrt{\kappa}}, \sqrt{\frac{1-\kappa}{2}}\right) \operatorname{dn}\left(\frac{t}{2 \kappa}, \kappa\right)+i \kappa \operatorname{sn}\left(\frac{t}{2 \kappa}, \kappa\right)}{\kappa \sqrt{2}\left[1-\sqrt{\frac{\kappa}{1+\kappa}} \operatorname{cn}\left(\frac{x}{\sqrt{\kappa}}, \sqrt{\frac{1-\kappa}{2}}\right) \operatorname{cn}\left(\frac{t}{2 \kappa}, \kappa\right)\right]},
$$

where $\kappa$ is again an arbitrary parameter (with $0<\kappa<1$ ).

It is again mentioned that all the above solutions refer to the integrable NLS system with $v=0$ (local nonlinearity) and, as such, the nonlocality cannot appear as part of these solutions. Below we will show that such solutions (and their generalizations) can be numerically extended, through the methodology discussed herein, for nonzero values of the nonlocality parameter.

\section{CONTINUATION TO THE NONLOCAL CASE}

\section{A. Peregrine soliton}

The results associated with the Peregrine soliton are given in Figs. 1 and 2. The first one shows the profile of the Peregrine soliton as the value of the nonlocality parameter $v$ is increased. Perhaps the most important finding in itself is that this structure can still be obtained as a numerically exact solution beyond the integrable limit, and in the nonlocal case of $v \neq 0$. Structurally, it can be observed that the solution acquires a certain "undulation," as $v$ is increased, that becomes progressively more pronounced. It is important to also highlight here that the solution is identified with periodic boundary conditions in both space and time (recall that time is treated as a space variable so a periodicity is imposed on that as well). The continuation scheme is unable to go past $v=0.2$, even when considering different domain sizes. Nevertheless, we do not detect a bifurcation at this point, hence it is unclear whether this is a trait of the solution or a byproduct of the particular numerical method. We believe that the latter may be true.

Figure 2, in turn, is a dynamical illustration of the accuracy of convergence of our solution. Here, what is done is that we select the "initial condition" of our converged Peregrine wave form at $t=-7.5$ and feed it into an integrator of the full nonlocal problem at different values of $v$. The forward propagation of Eqs. (1) and (2) is performed using the exponential time-differencing fourth-order Runge-Kutta (ETDRK4) method of Ref. [55]. In the figure, it is observed that the measured $L^{\infty}$ error (i.e., norm of the difference) between the converged solution and the numerically propagated one remains very small [i.e., of $\mathrm{O}\left(10^{-4}\right)$ to $\mathrm{O}\left(10^{-5}\right)$ ] throughout the propagation. We should factor in here the dynamical instability of the background of the solution, which eventually leads to growth (well past the formation and disappearance of the Peregrine soliton). An indirect manifestation of this origin of the observed growth can be seen in Fig. 2 in that the appearance and disappearance of the rogue wave does not seem to affect the exponential growth due to the dynamical instability of the background. Nevertheless, for all practical purposes, the converged state accurately captures the appearance and disappearance of the coherent structure.

\section{B. Kuznetsov-Ma breather}

In this subsection, we present similar diagnostics for the $\mathrm{KM}$ breather wave form of Eq. (5). It is interesting that, here, the background modulation becomes more transparent and

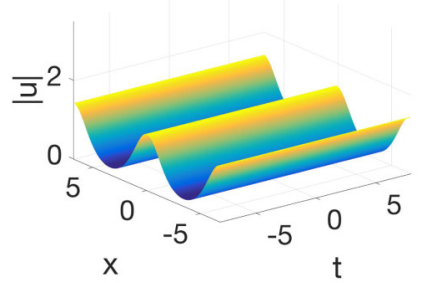

(a)

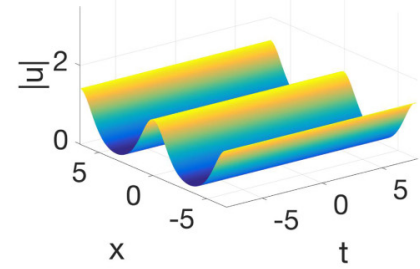

(b)

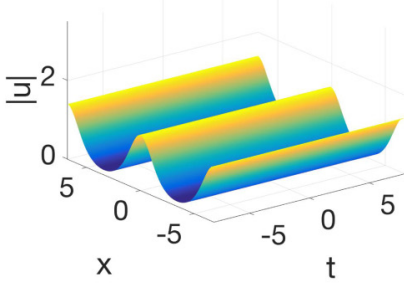

(c)

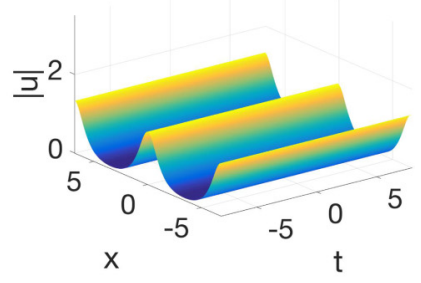

(d)

FIG. 6. Convergence to the stationary, periodic in space solution, obtained upon using as initial condition a point on the branch in Fig. 5, and decreasing $v$. This periodic solution apparently exists all the way to the local NLS limit. Values of $v$ used in each of the panels: (a) 0 , (b) 0.5 , (c) 1 , (d) 2 . 


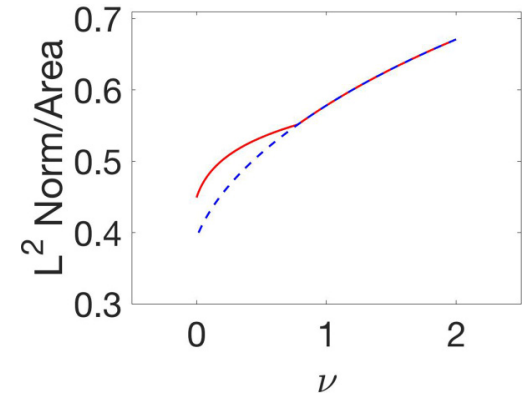

FIG. 7. Bifurcation diagram showing the doubly periodic solution (solid) and the periodic in space profile solution (dashed) as $v$ varies. Clearly, as $v$ increases, the two branches merge with each other, i.e., the periodic (in time) orbit emerges from the stationary solution.

arises in the clear form of a progressively more intense (as the nonlocality parameter $v$ increases) periodic background. The resulting wave forms at $v=0.15-0.2$ are strongly reminiscent of the rogue waves on a periodic (i.e., elliptic function) background recently discovered in integrable models such as the nonlinear Schrödinger [56] and the modified Korteweg-de Vries [57] models. While such solutions have not previously been found, to the best of our knowledge, in the nonlocal model these results are strongly suggestive that they exist. Whether they can be identified in this nonintegrable model in some closed form remains an outstanding problem for future study. We should note here that the KM wave form can only be continued up to around $v=0.25$, but not beyond that.

The verification of the accuracy of the numerical solution is given in Fig. 4, in a way similar to what was done before in
Fig. 2. Indeed, in this case the error up to $t=5$ does not grow in all the cases considered beyond $\mathrm{O}\left(10^{-6}\right)$. The associated growth observed in the ETDRK4 simulations initialized with the KM initial condition can be attributed to the exponential instability of the background seeded by the residual numerical error that eventually will grow to lead to deviations of $\mathrm{O}(1)$ at sufficiently long times (propagation distances).

\section{First doubly periodic solution}

We now turn to the doubly periodic solution Eq. (7) for which the results are presented in Figs. 5-8; here we have chosen $\kappa=0.8$. As can be seen in Fig. 5, the amplitude of the solution is initially doubly periodic in both space and time but gradually, as the nonlocality parameter is increased, becomes singly periodic in space. By this, we mean that the modulus of the solution becomes time independent (i.e., we exclude phase factors that can be eliminated by means of a gauge transformation).

This naturally suggests the question of whether this stationary, periodic in space solution of the nonlocal problem emerges only in the nonlocal model or exists in the local limit. Indeed, continuing the stationary solution "downward" (i.e., for decreasing $v$ ), one can see that it bifurcates from a stationary (elliptic function) solution at the local NLS limit, given explicitly by

$$
u=\exp \left(i \frac{\alpha t}{T}\right) A \operatorname{dn}\left(A x, \sqrt{2+\frac{2 \alpha-1}{A^{2}}}\right),
$$

where $\alpha=\frac{2 \pi}{T}$ and $A \approx 1.43$. The case examples of this state (for the same values of $v$ as in Fig. 5) are shown in Fig. 6.

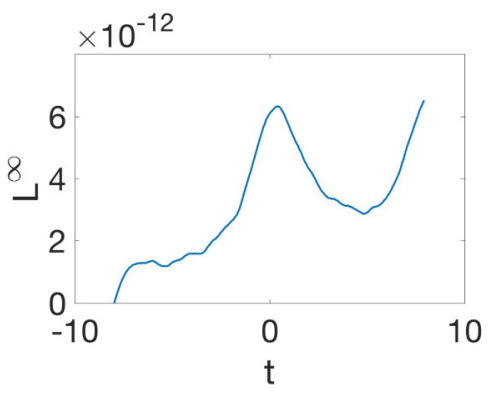

(a)

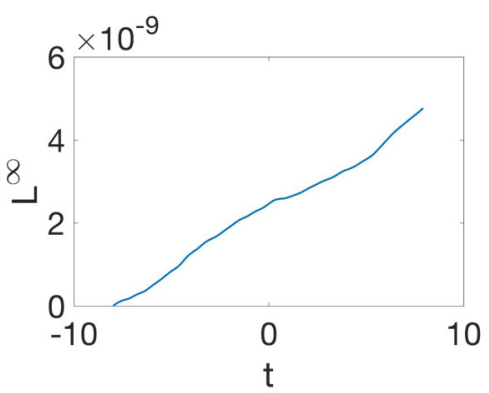

(d)

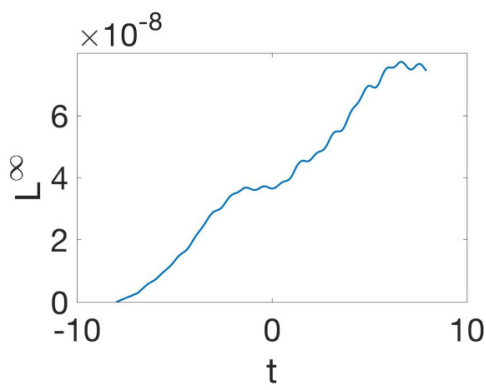

(b)

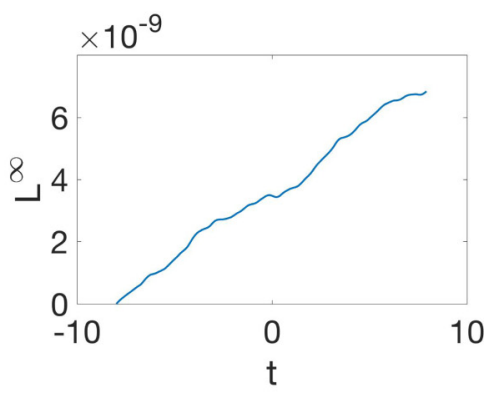

(e)



(c)

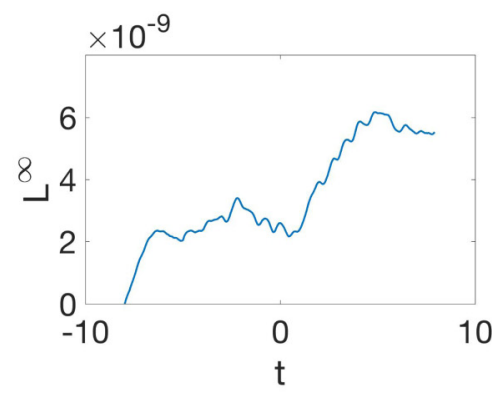

(f)

FIG. 8. $L^{\infty}$ error between the solution obtained by the Newton-CG method (as shown in Fig. 5) versus the solution obtained via the ETDRK4 method. Different values of $v$ up to $v=2$ are shown. Recall that in panels (c)-(f) the solution is, in fact, stationary. In panels (e) and (f), the error is shown for the solutions continued back down to lower values of $v$ along the branch of stationary (periodic in space) modulus. Values of $v$ used in each of the panels: (a) 0 , (b) 0.5 , (c) 1 , (d) 2, (e) 0.5 , (f) 0 . 


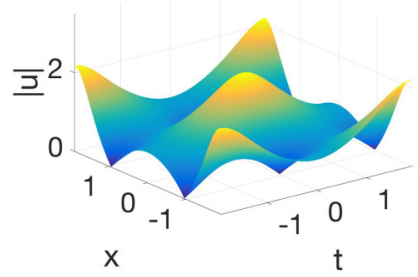

(a)

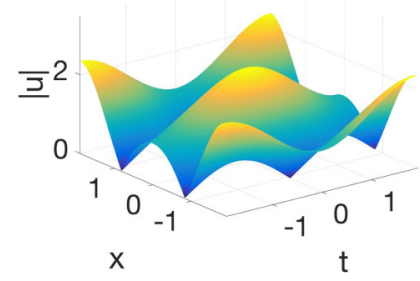

(b)

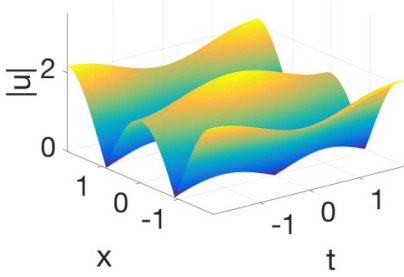

(c)



(d)

FIG. 9. Continuation over increasing $v$ values of the second doubly periodic solution of the NLS. It is seen that the doubly periodic solution bifurcates into a solution whose profile is constant in time as $v$ is increased. Values of $v$ used in each of the panels: (a) 0 , (b) 1.5 , (c) 3, (d) 6 .

Indeed, the relevant bifurcation diagram illustrating the merger of the space-time periodic solution with the spaceperiodic one is shown in Fig. 7. The emergence of a solution with a finite periodicity from a stationary one suggests a Hopf scenario in the Hamiltonian system at hand.

Lastly, here too, we examine the growth of the residual and find it to be very small $\left[\left(10^{-8}\right)\right.$ at the highest], when considering this class of solutions for different $v$ 's even up to $v=2$ (for which the solution is stationary). The relevant results are presented in Fig. 8 for the periodic solution in time (that turns stationary as $v$ is increased). We show prototypical examples for the space-time periodic solution in Figs. 8(a) and 8(b), then for higher values of $v$ where the wave form acquires a stationary modulus in Figs. 8(c) and 8(d). Also, for completeness, in Figs. 8(e) and 8(f) we go back down to lower values of $v$ along the branch of solutions of stationary modulus (i.e., periodic only in space) and examine the error in this case as well, confirming that it remains quite small during the evolution interval considered.

\section{Second doubly periodic solution}

In a similar vein, we now examine the continuation of the $v=0$ solution Eq. (8) to the nonlocal regime. We find a similar phenomenology as a result of the continuation as in the previous section. Namely, the doubly periodic solution, as $v$ is increased, turns to a singly periodic one in the case of sufficiently large $v$; see Fig. 9. On the other hand, starting at $v=2$ and continuing the stationary (modulus) solution down to $v=0$, we find that the relevant wave form exists for all $v$ down to the local limit, as illustrated in Fig. 10. That is to say, there is again a bifurcation diagram Fig. 11 illustrating the Hopf-type emergence of the periodic (in time) orbit from the stationary one (for decreasing $v$ ).

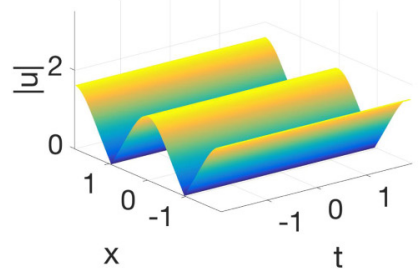

(a)



(b)

An additional interesting observation is that this stationary solution in the NLS limit $(v=0)$ degenerates to an explicit cnoidal solution in the form of

$$
u(x)=A \operatorname{cn}\left(\sqrt{-1+2 A^{2}} x, \sqrt{\frac{2 A^{2}}{2\left(-1+2 A^{2}\right)}}\right),
$$

with $A \approx 1.62$. Further, we can also arrive at an exact expression for the stationary state as $v \rightarrow \infty$. Inspired by the numerics, we can judiciously guess a solution of the form

$$
\begin{aligned}
& u=A \cos (B x) \\
& \theta=C \cos (D x)+E,
\end{aligned}
$$

and use it in Eq. (2). Doing so, and matching terms, one gets conditions on the constants, namely,

$$
B=\frac{D}{2}, \quad E=\frac{A^{2}}{2}, \quad C=\frac{\beta}{2} \frac{A^{2}}{D^{2}+\beta},
$$

where $\beta=\frac{2}{v}$. Consequently,

$$
\begin{aligned}
& u=A \cos \left(\frac{D}{2} x\right) \\
& \theta=\frac{\beta}{2} \frac{A^{2}}{D^{2}+\beta} \cos (D x)+\frac{A^{2}}{2} .
\end{aligned}
$$

Plugging this into the left-hand side of Eq. (1) and simplifying yields

$$
\begin{aligned}
i \frac{\partial u}{\partial t}+ & \frac{1}{2} \frac{\partial^{2} u}{\partial x^{2}}+\theta u-\frac{1}{2} u \\
= & -\left(\frac{D^{2}}{8}+\frac{1}{2}-\frac{A^{2}}{2}\right) A \cos \left(\frac{D}{2} x\right) \\
& +\left(\frac{\beta A^{2}}{2} \frac{A}{D^{2}+\beta}\right) \cos (D x) \cos \left(\frac{D}{2} x\right) .
\end{aligned}
$$

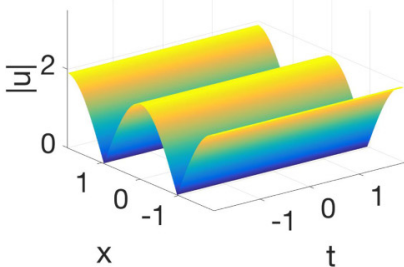

(c)

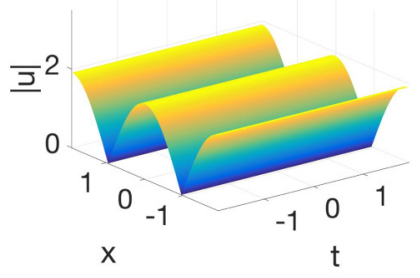

(d)

FIG. 10. Using as initial condition the solution in Fig. 9(d), we decrease $v$ and find the continuation of the stationary branch down to the NLS limit of $v=0$. Values of $v$ used in each of the panels: (a) 0 , (b) 1.5 , (c) 3, (d) 6 . 


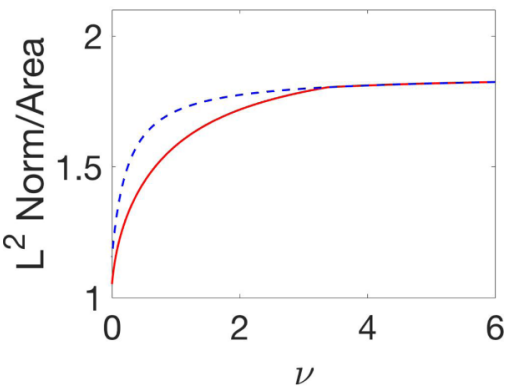

FIG. 11. Bifurcation diagram showing the second doubly periodic solution (solid) and the constant profile solution (dashed) as $v$ varies.

Taking $\beta \rightarrow 0$, or equivalently $\nu \rightarrow \infty$, the above reduces to $-\left(\frac{D^{2}}{8}+\frac{1}{2}-\frac{A^{2}}{2}\right) A \cos \left(\frac{D}{2} x\right)$, which we can make zero provided we choose

$$
\frac{D^{2}}{8}+\frac{1}{2}-\frac{A^{2}}{2}=0
$$

This has two consequences. The first is that, as $v \rightarrow \infty$, we have the exact solution:

$$
\begin{aligned}
& u=A \cos \left(\sqrt{A^{2}-1} x\right) \\
& \theta=\frac{A^{2}}{2} .
\end{aligned}
$$

The second, related one, is that under the limit $v \rightarrow \infty$, the nonlocal NLS can lead to the following linear Schrödinger equation:

$$
\frac{1}{2} \frac{\partial^{2} u}{\partial x^{2}}+\frac{A^{2}}{2} u-\frac{1}{2} u=0 .
$$

From the bifurcation diagram in Fig. 11, as $v$ increases, we have found that the amplitude of $u$ approaches the constant value of $A \approx 1.92$.

It is relevant to remark here that the numerically obtained periodic in space solution is well approximated by the above asymptotic functional form. At $v=6$, the pointwise error between the two is approximately $2 \times 10^{-2}$. In fact, our numerical observations suggest that one can approximate the numerically obtained periodic in space solution by the functional form,

$$
u=A \operatorname{cn}(B x, C),
$$

for suitably chosen $A, B, C$. In fact, with this approximation, the pointwise error can be made between $10^{-3}$ and $10^{-6}$ for all $v$ in the interval $0 \leqslant v \leqslant 6$. This suggests the possibility of seeking suitable elliptic function solutions as potentially exact wave forms of the nonlocal model. This merits a separate investigation beyond the confines of the present paper. Lastly, we note that for both branches of solutions, we present the diagnostic of the $L^{\infty}$ norm of the deviation from the numerically identified solution when propagating the corresponding initial condition with ETDRK4 in Fig. 12. This is similar to Fig. 8 with the top panels pertaining to the space-time periodic branch, while the bottom ones arise for the stationary modulus, periodic in space branch present for large $v$, but which can be continued down to lower values of $v$. Once again, the relevant residual grows due to the instability of the

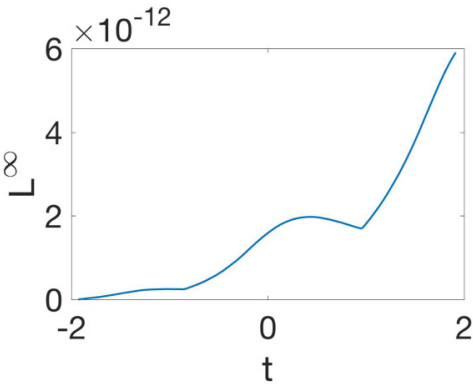

(a)

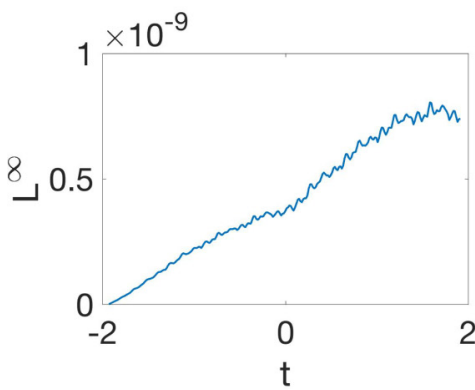

(d)

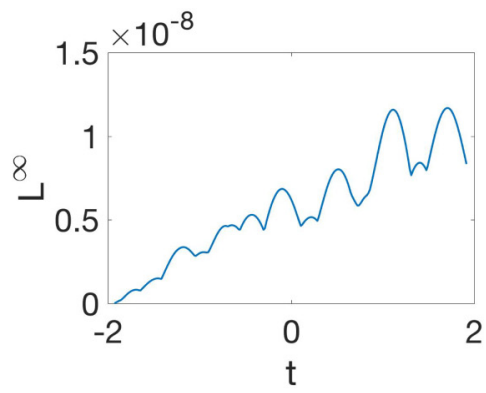

(b)

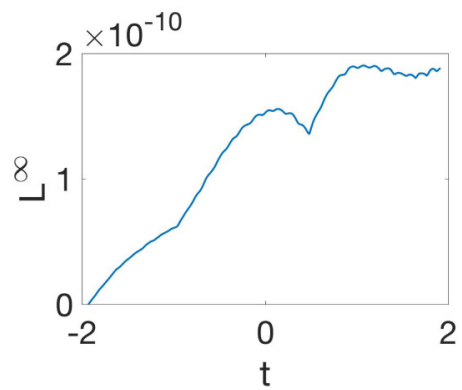

(e)

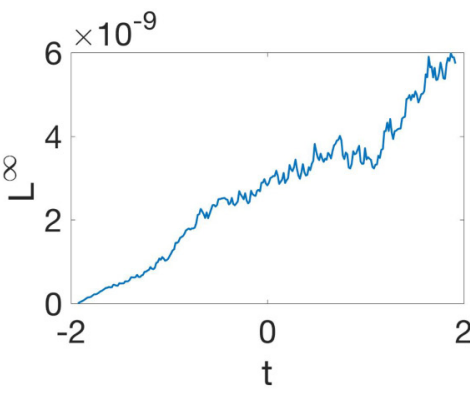

(c)

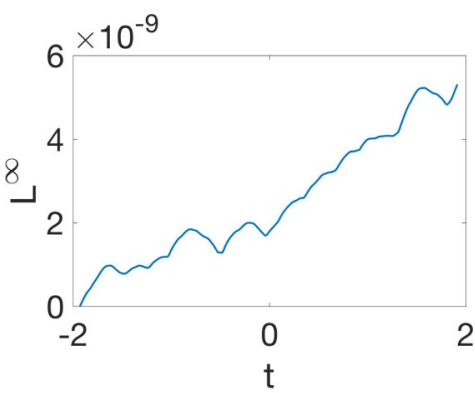

(f)

FIG. 12. $L^{\infty}$ error between the solution obtained by the Newton-CG method (as shown in Fig. 9) versus the solution obtained via the ETDRK4 method. The three top panels correspond to the error of the space time periodic solution, while the bottom three case examples are examples of the error in the evolution of wave forms of the stationary modulus branch. Values of $v$ used in each of the panels: (a) 0 , (b) 1.5 , (c) 3 , (d) 6 , (e) 1.5 , (f) 0 . 
background, yet remains bounded by $10^{-8}$ over the timescales considered.

\section{CONCLUSIONS AND FUTURE WORK}

In the present paper, we considered a variety of wave structures existing in the local NLS equation and extended them to the realm of a generic nonlocal NLS model. In this vein, we examined the prototypical rogue wave state, namely the Peregrine soliton, as well as its periodic in time generalization, the KM breather. We also looked at other states that are periodic in both space and time, motivated by the work of Ref. [54]. These continuations led to a number of interesting conclusions. Both the Peregrine soliton and the $\mathrm{KM}$ breather were possible to continue for small values of the nonlocality parameter $v$. This is appealing because it suggests that the structures are not particular to the integrable limit and can, indeed, be continued in the nonintegrable case. Additionally, as the structures are continued, they develop undulations which, in some cases (e.g., the KM state), suggest connections with other states that have been recently identified in integrable models, namely, rogue waves mounted on elliptic function, spatially periodic structures. With regard to the doubly periodic states with periodicities in both space and time, these were more robustly identified through our continuation scheme and could, in fact, be continued up to $v=2$ and beyond. However, here too, there exists an interesting twist, namely, the structures beyond a certain degree of nonlocality lost their temporal periodicity and became genuinely stationary in their modulus, maintaining only the spatial periodicity. These spatially periodic states were subsequently continued downward all the way to the local NLS limit, confirming their cnoidal nature in the latter and revealing the bifurcation of their spatiotemporally periodic counterparts.

We believe that these results offer considerable insight into the potential of the nonlocal model to support states (including rogue wave ones) with nonvanishing asymptotics, i.e., ones beyond the more "standard" solitary wave ones. However, additionally, they also motivate a number of further questions and inquiries worth considering in future studies. One of these is whether stationary elliptic function solutions can be suitably generalized in analytically available wave forms in the nonlocal case (or whether these can only be identified in limiting cases such as the ones of $v \rightarrow 0$ and $v \rightarrow \infty$ considered herein). Another topic of interest is to systematically explore continuations of the rogue waves on periodic wave background and explore how such structures may generalize in the case of the nonlocal model. Possibly, these may be involved in bifurcation phenomena associated with the states considered here. Another more open ended challenge is whether rogue-wave-like patterns, such as the Peregrine soliton or the KM breather, can be continued beyond the intervals of $v$ for which they were found herein in the case of the nonlocal model. Lastly, in the present paper, we focused chiefly on the existence and in some cases on the bifurcations of the solutions. However, there are stability tools gradually emerging (such as, e.g., the Floquet analysis of the KM state and the consideration of the Peregrine as a limiting case of that calculation [58]) that would be quite relevant to consider in the present nonlocal setting as well. While there is no direct, universally accepted notion of stability of rogue wave structures such as the Peregrine (hence, we have not extended our considerations to this direction herein), such tools are worth examining aiming to offer some initial insights toward the study of stability. Potential progress in any of these directions will be reported in future publications.

\section{ACKNOWLEDGMENTS}

P.G.K. acknowledges support from the US National Science Foundation under Grants No. PHY-1602994 and No. DMS-1809074. He also acknowledges support from the Leverhulme Trust via a Visiting Fellowship and the Mathematical Institute of the University of Oxford for its hospitality during part of this work.
[1] W. Krolikowski, O. Bang, N. I. Nikolov, D. Neshev, J. Wyller, J. J. Rasmussen, and D. Edmundson, J. Opt. B: Quantum Semiclass. Opt. 6, S288 (2004).

[2] G. Assanto, A. A. Minzoni, and N. F. Smyth, J. Nonlin. Opt. Phys. Mat. 18, 657 (2009).

[3] G. Assanto, Nematicons: Spatial Optical Solitons in Nematic Liquid Crystals (Wiley-Blackwell, New Jersey, 2012).

[4] C. Rotschild, O. Cohen, O. Manela, M. Segev, and T. Carmon, Phys. Rev. Lett. 95, 213904 (2005).

[5] C. Conti, M. Peccianti, and G. Assanto, Phys. Rev. Lett. 91, 073901 (2003).

[6] A. G. Litvak, V. A. Mironov, G. M. Fraiman, and A. D. Yunakovskii, Sov. J. Plasma Phys. 1, 60 (1975).

[7] A. I. Yakimenko, Y. A. Zaliznyak, and Y. S. Kivshar, Phys. Rev. E 71, 065603(R) (2005).

[8] T. P. Horikis and D. J. Frantzeskakis, Phys. Rev. Lett. 118, 243903 (2017).

[9] T. P. Horikis and D. J. Frantzeskakis, Proc. Roy. Soc. A 475, 20190110 (2019).
[10] P. Pedri and L. Santos, Phys. Rev. Lett. 95, 200404 (2005).

[11] M. J. Edmonds, T. Bland, D. H. J. O’Dell, and N. G. Parker, Phys. Rev. A 93, 063617 (2016).

[12] N. I. Nikolov, D. Neshev, O. Bang, and W. Z. Krolikowski, Phys. Rev. E 68, 036614 (2003).

[13] M. Bache, O. Bang, J. Moses, and F. W. Wise, Opt. Lett. 32, 2490 (2007).

[14] M. Bache, O. Bang, W. Krolikowski, J. Moses, and F. W. Wise, Opt. Express 16, 3273 (2008).

[15] P. V. Larsen, M. P. Sørensen, O. Bang, W. Z. Królikowski, and S. Trillo, Phys. Rev. E 73, 036614 (2006).

[16] A. Dreischuh, D. N. Neshev, D. E. Petersen, O. Bang, and W. Krolikowski, Phys. Rev. Lett. 96, 043901 (2006).

[17] M. Shen, J.-J. Zheng, Q. Kong, Y.-Y. Lin, C.-C. Jeng, R.-K. Lee, and W. Krolikowski, Phys. Rev. A 86, 013827 (2012).

[18] B. K. Esbensen, M. Bache, O. Bang, and W. Krolikowski, Phys. Rev. A 86, 033838 (2012).

[19] N. Akhmediev, A. Ankiewicz, and M. Taki, Phys. Lett. A 373, 675 (2009). 
[20] O. Bang and M. Peyrard, Phys. Rev. E 53, 4143 (1996).

[21] M. H. Frosz, O. Bang, and A. Bjarklev, Opt. Express 14, 9391 (2006).

[22] A. Chabchoub, N. P. Hoffmann, and N. Akhmediev, Phys. Rev. Lett. 106, 204502 (2011).

[23] A. Chabchoub, N. Hoffmann, M. Onorato, and N. Akhmediev, Phys. Rev. X 2, 011015 (2012).

[24] A. Chabchoub and M. Fink, Phys. Rev. Lett. 112, 124101 (2014).

[25] D. R. Solli, C. Ropers, P. Koonath, and B. Jalali, Nature 450, 1054 (2007).

[26] B. Kibler, J. Fatome, C. Finot, G. Millot, F. Dias, G. Genty, N. Akhmediev, and J. M. Dudley, Nat. Phys. 6, 790 (2010).

[27] B. Kibler, J. Fatome, C. Finot, G. Millot, G. Genty, B. Wetzel, N. Akhmediev, F. Dias, and J. M. Dudley, Sci. Rep. 2, 463 (2012).

[28] J. M. Dudley, F. Dias, M. Erkintalo, and G. Genty, Nat. Photon. 8, 755 (2014).

[29] B. Frisquet, B. Kibler, P. Morin, F. Baronio, M. Conforti, G. Millon, and S. Wabnitz, Sci. Rep. 6, 20785 (2016).

[30] C. Lecaplain, Ph. Grelu, J. M. Soto-Crespo, and N. Akhmediev, Phys. Rev. Lett. 108, 233901 (2012).

[31] G. Genty, C. M. de Sterke, O. Bang, F. Dias, N. Akhmediev, and J. M. Dudley, Phys. Lett. A 374, 989 (2010).

[32] A. N. Ganshin, V. B. Efimov, G. V. Kolmakov, L. P. MezhovDeglin, and P. V. E. McClintock, Phys. Rev. Lett. 101, 065303 (2008).

[33] H. Bailung, S. K. Sharma, and Y. Nakamura, Phys. Rev. Lett. 107, 255005 (2011)

[34] M. Onorato, S. Residori, U. Bortolozzo, A. Montina, and F. T. Arecchi, Phys. Rep. 528, 47 (2013).

[35] P. T. S. DeVore, D. R. Solli, D. Borlaug, C. Ropers, and B. Jalali, J. Opt. 15, 064001 (2013).

[36] Z. Yan, J. Phys. Conf. Ser. 400, 012084 (2012).

[37] N. Akhmediev, B. Kibler, F. Baronio, M. Belić, W.-P. Zhong, Y. Zhang, W. Chang, J. M. Soto-Crespo, P. Vouzas, P. Grelu et al., J. Opt. 18, 063001 (2016).
[38] S. Chen, F. Baronio, J. M. Soto-Crespo, P. Grelu, and D. Mihalache, J. Phys. A: Math. Theor. 50, 463001 (2017).

[39] D. Mihalache, Rom. Rep. Phys. 69, 403 (2017).

[40] B. A. Malomed and D. Mihalache, Rom. J. Phys. 64, 106 (2019).

[41] Extreme Ocean Waves, edited by E. Pelinovsky and C. Kharif (Springer-Verlag, New York, 2008).

[42] C. Kharif, E. Pelinovsky, and A. Slunyaev, Rogue Waves in the Ocean (Springer-Verlag, New York, 2009).

[43] A. R. Osborne, Nonlinear Ocean Waves and the Inverse Scattering Transform (Academic Press, Amsterdam, 2010).

[44] M. Onorato, S. Residori, and F. Baronio, Rogue and Shock Waves in Nonlinear Dispersive Media (Springer-Verlag, Heidelberg, 2016).

[45] B. Yang and J. Yang, Lett. Math. Phys. 109, 945 (2019).

[46] M. J. Ablowitz and Z. H. Musslimani, Phys. Rev. Lett. 110, 064105 (2013).

[47] T. P. Horikis and M. J. Ablowitz, Phys. Rev. E 95, 042211 (2017).

[48] C. B. Ward, P. G. Kevrekidis, and N. Whitaker, Phys. Lett. A 383, 2584 (2019).

[49] B. K. Esbensen, M. Bache, W. Krolikowski, and O. Bang, Phys. Rev. A 86, 023849 (2012).

[50] J. Wyller, W. Krolikowski, O. Bang, and J. J. Rasmussen, Phys. Rev. E 66, 066615 (2002).

[51] D. H. Peregrine, J. Austral. Math. Soc. B 25, 16 (1983).

[52] E. A. Kuznetsov, Sov. Phys.-Dokl. 22, 507 (1977).

[53] Ya. C. Ma, Stud. Appl. Math. 60, 43 (1979).

[54] N. N. Akhmediev, V. M. Eleonskii, and N. E. Kulagin, Theor. Math. Phys. 72, 809 (1987).

[55] A.-K. Kassam and L. N. Trefethen, SIAM J. Sci. Comput. 26, 1214 (2005).

[56] J. Chen, D. E. Pelinovsky, and R. E. White, arXiv:1905.11638.

[57] J. Chen and D. E. Pelinovsky, J. Nonlinear Sci. 29, 2797 (2019).

[58] J. Cuevas-Maraver, P. G. Kevrekidis, D. J. Frantzeskakis, N. I. Karachalios, M. Haragus, and G. James, Phys. Rev. E 96, 012202 (2017). 